\title{
Assessment of certain applications of Vehicle-to-Vehicle communication in an urban network
}

\author{
A. G. Hobeika \& T. Kim \\ Department of Civil and Environmental Engineering, \\ Virginia Polytechnic Institute and State University, USA
}

\begin{abstract}
The information obtained from Connected Vehicles has the potential of providing local and area-wide traffic management solutions, which is desperately needed in most large urban areas. The reliability and performance of this transmitted information have to be addressed to ensure that the users can properly utilize this information to solve traffic management issues. This paper investigates the success rate of wireless communication and area covered in space and time by Vehicle-to-Vehicle communication in an urban network based on different market penetration rates and wireless communication coverage. Results show that both average success rate of communication and area coverage level increase as the market penetration rate and wireless communication coverage increase. At least $90 \%$ of equipped vehicles are successful in communicating with nearby equipped vehicles in a Whole Network case. The market penetration rate of equipped vehicles has a greater influence on spatialtemporal dispersion of equipped vehicles. The Selected Links case has higher average area coverage level than the Whole Network case although it has lower success rate of communication. Finally, the spatial-temporal dispersion of equipped vehicles increase as the density of traffic increases, i.e. more equipped vehicles are able to communicate with one another. These study findings will be useful for making decisions about investments in cooperative vehicles in relation to the expected increase in traffic efficiency.
\end{abstract}

Keywords: Connected Vehicle, Vehicle-to-Vehicle (V2V), vehicle wireless communication, area coverage, TRANSIMS. 


\section{Introduction}

Connected Vehicle research has emerged as one of the highest priorities in the transportation field. It focuses on Vehicle-to-Vehicle (V2V) and Vehicle-toInfrastructure (V2I) communication using wireless technologies such as Dedicated Short Range Communications (DSRC). Most of the automakers and a number of countries are developing Connected Vehicle applications. The intent is to support safety, mobility, and environmental applications that benefit the users and providers of the transportation system. Connected Vehicles can provide continuous real-time information to all system users including vehicles, infrastructure, wireless devices, and transportation management centres. V2V communication enables an equipped vehicle to avoid crashes by receiving warning threats or hazards. V2I wireless communication enables an equipped vehicle to exchange safety and operational data with highway infrastructure.

The information obtained from Connected Vehicles has the potential of providing local and area-wide traffic management solutions which is desperately needed in most large urban areas. The reliability and the frequency of this transmitted information have to be addressed to ensure that the users can properly utilize this information to solve traffic management issues. This paper focuses on the area covered in space and in time by V2V communication in an urban network based on different market penetration rates and wireless communication coverage.

\section{Literature review}

The literature covers a wide range of topics in the Connected Vehicle research. It is ubiquitous and is not possible to cite it all in this condensed paper. However, the authors will focus on selected publications that cover the areas of communications and standards and on issues related to penetration levels of Connected Vehicles and related research work conducted on probe vehicles and the requirements needed to obtain reliable information for traffic management purposes.

Many countries are working on developing DSRC technology for various connected vehicle applications to improve safety and mobility. The wireless communication coverage is one of the important factors for connected vehicle functionalities. But, it is difficult to evaluate wireless vehicular communication coverage at each different site because it depends on various factors that may obstruct the vehicular communication. Gallagher et al. [1] implemented a field test for line-of-sight (LOS) and non-line-of-sight (NLOS) radio links for $5.9 \mathrm{GHz}$ DSRC. The results showed that the maximum highway $\mathrm{V} 2 \mathrm{~V}$ range is $880 \mathrm{~m}$ (2886 feet) with for LOS when there is no obstruction in the field. Also, the V2V range for NLOS is from 59 to $230 \mathrm{~m}$. Meireles et al. [2] studied the effect of vehicles as physical obstructions for the wireless signal using two cars equipped with DSRC. They performed experimental measurements to collect received signal power and packet delivery ratio information in a multitude of relevant scenarios: parking lot, highway, suburban and urban canyon. The results showed 
that obstructing vehicles can cause significant impact on the channel quality. ASTM E2213-03, one of standards for DSRC, indicates that the communications generally occur over line-of-sight distances of less than 1000 meters for V2I or V2V [3]. IEEE 1609 is another standard for Wireless Access in Vehicular Environments (WAVE). WAVE standard also mention that wireless communications occur up to $1000 \mathrm{~m}$ in the vehicular environment [4].

Studies on market penetration rate for $\mathrm{V} 2 \mathrm{~V}$ are discussed next. Market penetration is required for the potential deployment of new application or service. Shladover et al. [5] studied a performance of cooperative vehicle systems (CVS) based on the market penetration of equipped vehicles utilizing Monte Carlo analyses and simulations of wireless message propagation. The effectiveness of both direct and transport relay modes were tested as a function of wireless communication range, market penetration, and traffic density and their influence on the speed of message propagation. The results indicated that the direct relay is more effective in a high density of equipped vehicles. But, the relays became more dependent on the transport relay in a low density of equipped vehicles. Cheol Oh et al. [6] presented an enhanced traffic surveillance system capable of collecting probe vehicle information using GPS and V2V communications by different $\mathrm{V} 2 \mathrm{~V}$ communication range, market penetration rate of equipped vehicles, and an aggregation interval for travel time estimation. The evaluation results showed that travel time errors of less than $5 \%$ and $15 \%$ are achievable under normal and incident traffic conditions, respectively. Mei et al. [7] described a simulation model for studying the impact of V2V communications on traffic network operations. The developed simulation model was used in a case study application to simulate vehicle dynamic route diversion and variable speed limits following a severe incident in a small network. Simulation results indicated that the model results are sensitive both to different market penetration levels of vehicles equipped with wireless communications capabilities and to various control strategies. Park and Lee [8] examined the sustainability impacts of route guidance system under the cooperative vehicle infrastructure environment using various market penetration rates of equipped vehicles. The simulation results indicated that the route guidance system under the cooperative vehicle infrastructure environment improves air qualities and fuel consumption by 10 to $18 \%$ compared to base case.

Area coverage is an important measurement to determine the required market penetration rate or sample size for probe vehicles. Ygnace et al. [9] developed an area coverage equation for probe vehicles used in San Francisco to estimate travel times as follows:

$$
\mathrm{E}=1-\exp (-\alpha \rho \mathrm{L})
$$

where $\mathrm{E}$ is the area coverage, $\alpha$ is the fraction of vehicles sampled, $\rho$ is the density of traffic per unit length, and $\mathrm{L}$ is the average link length. This equation translates that the fraction of vehicles sampled or average link length should be increased to improve the coverage. But, this model did not consider the location error or the link type. Srinivasan and Jovanis [10] developed a heuristic 
algorithm for determining number of probe vehicles required in urban network for reliability of travel time estimation and adequacy of area coverage. This study indicated a greater proportion of freeway links than of major arterials can reliably be covered with a given number of probes. Also, the number of probes required increases as the desired proportion of link coverage in the network increases. BMW Group [11] considered the potential of vehicle-generated traffic data acquisition for the generation of traffic information and local hazard warnings. A method for estimating the required floating car penetration rates on the basis of traffic volume and arrival probabilities is presented. This study showed the necessary penetration rates for different road network categories. For example, at least $10 \%$ of penetration rate is required for federal roads and urban arterials in peak hours.

The use of probe vehicle is an efficient method to collect real-time traffic information. Some studies are conducted to determine the number of probe vehicles to ensure acceptable data qualities for traffic information services. Turner and Holdener [12] investigated a statistical sampling method to obtain the minimum number of probe vehicles for obtaining real-time travel speeds and travel times in Houston. The minimum number of probe vehicles was calculated by two separate statistical scenarios and the current number of probe vehicles from AVI tags in Houston provides reliable travel speed information with 95\% confidence. Ygnace et al. [9] evaluated the feasibility of using cell phones as traffic probes to estimate travel time in the San Francisco Bay Area network. This study concluded that at least $5 \%$ of travellers on freeways that are equipped with a cell phone must be sampled for the accurate estimation of travel time. Green et al. [13] investigated the dynamic determination of sample sizes for traffic condition monitoring systems. This study used the central limit theorem (CLT) to estimate mean speed for 12 sites in Virginia. Some sites could be satisfied with $95 \%$ confidence interval with accuracy of $\pm 5 \mathrm{mph}$ from the sample size which has less than $5 \%$ of traffic.

Area coverage is an important measurement to determine the required market penetration rate. Although some previous studies on area coverage were conducted, they were mainly hypothetical and didn't use realistic and large networks. They used a cumulative area coverage level by short time durations such as 5, 10 and 15 minutes and low penetration rate such as $5 \%$. To obtain much more fine grained area coverage level from shorter time interval, such as 1 minute and 1 second intervals, with higher time duration should be determined to get better real-time information service.

\section{Research background}

\subsection{Objectives}

The objectives of this research are as follows;

1) Identify valid communication among the identified equipped vehicles in a realistic and large network, 2) determine the success rate of wireless communication among the identified equipped vehicles based on various market 
penetration rates, 3 ) determine the dispersion of equipped vehicles in time and in space in an urban network, and 4) calculate the area coverage obtained by the valid communication among equipped vehicles.

\subsection{Overview of TRANSIMS}

To assess the dispersion of equipped vehicles in time and in space requires a special simulation that can trace each individual vehicle in an urban area on a second by second basis and be able to carry the identity of each driver and passenger with it at all time. The simulation that can execute these requirements is TRANSIMS (Transportation Analysis and Simulation System) which is developed by Federal Highway Administration (FHWA), USA and is available in the public domain. The underlying TRANSIMS philosophy is that to study the transportation system's performance effectively, one needs to simulate travel in a study area with a rather fine temporal and spatial resolution. Other research and developmental efforts have also come to the conclusion that the next generation of urban travel models should be based on micro-analytic simulation and that they should employ the activity-based approach for modeling travel demand.

TRANSIMS differs from current travel demand forecasting methods in its underlying concepts and structure. These differences include a consistent and continuous representation of time; a detailed representation of persons and households; time-dependent routing; and a person-based microsimulator. Microsimulator is the only simulation tool that maintains the identity of the traveler throughout the simulation, and is capable of accessing the database of each individual (e.g., income, age, trip purpose, etc.). In other words, it traces the movement of people as well as vehicles on a second-by-second basis.

The unique features of TRANSIMS would allow the analyst to assess the dispersion of equipped vehicles by time and location in an urban area. There is no limit on the size of the urban area that can be simulated. In addition this system is able to include a detailed highway network such as the collector and the feeder systems. The equipped vehicles would be assigned to travelers in the Population Synthesizer Module based on their socio-economic characteristics. TRANSIMS also can map the location of all vehicles in the network at every second. The transmission power of the equipped vehicles can be varied and used to create contours of vehicle to vehicle coverage, hence giving the analyst the ability to assess the coverage level and the frequency of the information obtained from the equipped vehicles for different penetration scenarios, and determines the utility of the investment for different vehicle systems deployment levels.

\section{Research methodology}

\subsection{Overview of study process}

The city of Alexandria, VA, USA outside of Washington D.C. was used for this study. The size of study area is around $44 \mathrm{~km}^{2}\left(17 \mathrm{mi}^{2}\right)$, bordered by I-395 (Henry G Shirley Memorial Highway), I-495 (Capital Beltway), and State Route 400 as shown in Figure 1. TRANSIMS provided the dataset for Alexandria as 
the test case. This dataset includes all the input data needed to represent and code the highway and transit networks. This highway network has 3653 links and about 420,000 trips.

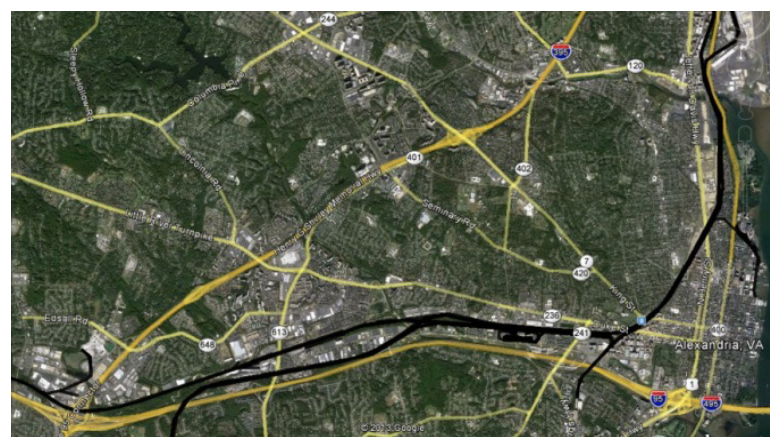

Figure 1: Alexandria study area.

Vehicles having a specified vehicle type and subtype in TRANSIMS are assumed as the equipped vehicles that can communicate with nearby other equipped vehicles to exchange traffic and travel information among each other. The fraction of equipped vehicles to total vehicles can be changed in the population synthesizer module of TRANSIMS to represent different market penetration rates. The population synthesizer module assigns vehicles including equipped vehicles to each household according to their socio-economic characteristics $[14,15]$. Then, the equipped and non-equipped vehicles are distributed onto the network through the modules of activity generator, router planner, and microsimulator. Figure 2 shows the flowchart of the study process.

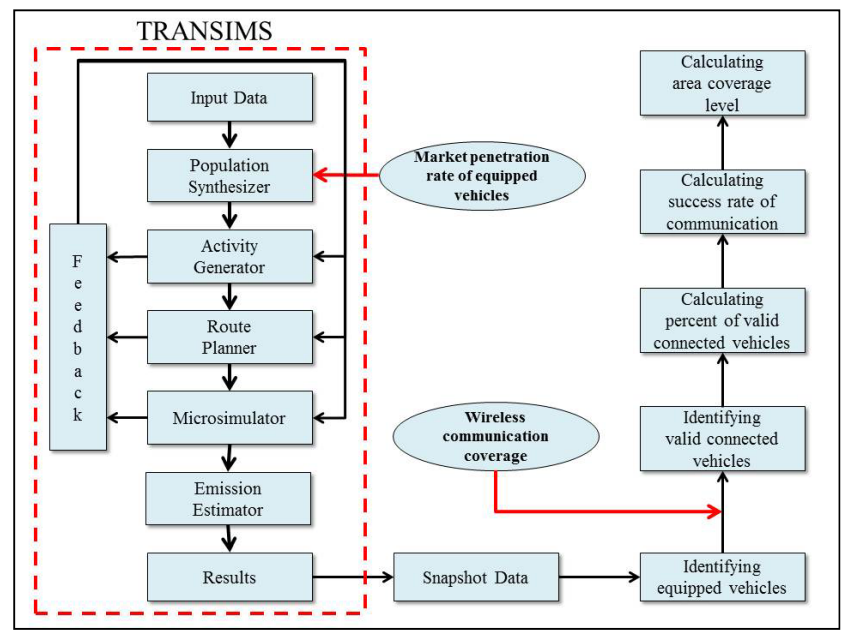

Figure 2: Study process flowchart. 
Each individual vehicle trajectory data are required to assess the dispersion of equipped vehicles in time and space in the study area. TRANSIMS offers detailed snapshot data as one of the output files from the microsimulator. This snapshot data include the location of each individual traveller, car, or transit vehicle on a second-by-second basis. Each equipped vehicle can be traced from the provided snapshot data output such as vehicle ID, link ID, travel direction, lane, vehicle type, speed, $\mathrm{x}, \mathrm{y}$, and $\mathrm{z}$ coordinates of each vehicle at specified time points. This study assumed a valid connected vehicle as the equipped vehicle that is able to communicate with other equipped vehicle when they are spatially within defined wireless communication coverage distance. After identifying all equipped vehicles using vehicle ID and vehicle type defined in population synthesizer module, the distances between all equipped vehicles are calculated using $\mathrm{x}$ and $\mathrm{y}$ coordinates from snapshot data. The percentage of valid connected vehicles, the success rate of communication among equipped vehicles, and the area coverage that they produce, would be used to assess the coverage level and the frequency of the information obtained from the equipped vehicles for different market penetration rates of equipped vehicles and wireless communication coverage distances.

\subsection{Scenarios}

This study considers two networks within the study area; the whole network and the selected links network. Two freeways, one expressway, and four major arterials are identified to compose the selected links network case in Alexandria while the whole network case covers all the links in the Alexandria network. The selected links network represents the higher functional class of the roadways which traditionally carries more than $70 \%$ of all travel at any time of the day and is worthy of studying it as a separate case. Nine scenarios are analyzed in this study based on two variables, market penetration rate of equipped vehicles and wireless communication coverage. Three market penetration rates of equipped vehicles $(10 \%, 20 \%$, and $40 \%)$, and three wireless communication coverage (100 $\mathrm{m}, 200 \mathrm{~m}$, and $500 \mathrm{~m}$ ) are evaluated for the two study area networks. In this study the maximum wireless communication coverage is limited to $500 \mathrm{~m}$ because of building and vehicular obstructions in the Alexandria network.

\section{Results}

\subsection{Average success rate of communication}

Several TRANSIMS runs were conducted to utilize the Alexandria data sets provided by FHWA. The vehicle data was collected from the snapshot output file at every one minute during 4 hours from 6 to $10 \mathrm{a} . \mathrm{m}$. Due to the huge size of the snapshot data file, the aggregation to the 1 minute level was needed in order to manage the large data for the four hours during the morning peak period. The total number of vehicles in the whole network is 105,470 vehicles for four hours. The one second movement of these vehicles for four hours would have created around 1.2 trillion data points for only one output variable, which would be hard 
to manage for multiple variables without utilizing super computers. The authors did organize the data at 10 seconds interval level for a 30 minutes period in order to gauge the differences in results.

The results of the average percentage of valid connected vehicle by three market penetration rates of equipped vehicles $(10,20$, and $40 \%)$ and three wireless communication coverage $(100,200$, and $500 \mathrm{~m})$ are shown in Table 1. The average percentage of valid connected vehicles to total vehicles in the study area is calculated to assess the strength of communication among $\mathrm{V} 2 \mathrm{~V}$ in an urban network. The actual market penetration rate of equipped vehicles obtained from snapshot data during the analysis period $(6-10$ a.m.) is less than the designated market penetration rate, which is calculated as a percent of the total daily vehicles to be assigned onto the network. The average percentage of valid connected vehicles increases as market penetration rate of equipped vehicles and wireless communication coverage increase as shown in Table 1 . The average percentage of valid connected vehicles is more sensitive to the market penetration rate of equipped vehicles than the range of communication coverage. The increase in wireless communication coverage from $100 \mathrm{~m}$ to $500 \mathrm{~m}$ for the same daily market penetration rate of $10 \%$ of equipped vehicles only produced an increase of 0.59 percent of valid connected vehicles. While, an increase of daily market penetration rate from $10 \%$ to $20 \%$ produced a $6.11 \%$ increase in valid connected vehicles. The conclusion is that market penetration rate has a greater influence on the validity of connected vehicles than communication area coverage.

Table 1: Average percentage of valid connected vehicle.

\begin{tabular}{|c|c|c|c|c|c|c|c|}
\hline \multirow{3}{*}{\multicolumn{2}{|c|}{$\begin{array}{l}\text { Market penetration rate of } \\
\text { equipped vehicles over } 20 \text {-hours }\end{array}$}} & \multicolumn{6}{|c|}{ Study area } \\
\hline & & \multicolumn{3}{|c|}{ Whole Network } & \multicolumn{3}{|c|}{ Selected Links } \\
\hline & & $10 \%$ & $20 \%$ & $40 \%$ & $10 \%$ & $20 \%$ & $40 \%$ \\
\hline \multicolumn{2}{|c|}{$\begin{array}{l}\text { Actual market penetration rates } \\
\text { of equipped vehicles over } \\
4 \text { hours }\end{array}$} & $6.25 \%$ & $12.32 \%$ & $24.42 \%$ & $7.02 \%$ & $13.80 \%$ & $27.45 \%$ \\
\hline \multirow{3}{*}{$\begin{array}{l}\text { Wireless } \\
\text { Communication } \\
\text { Coverage }\end{array}$} & $100 \mathrm{~m}$ & $5.63 \%$ & $11.74 \%$ & $23.88 \%$ & $5.40 \%$ & $12.31 \%$ & $25.99 \%$ \\
\hline & $200 \mathrm{~m}$ & $6.04 \%$ & $12.12 \%$ & $24.24 \%$ & $6.28 \%$ & $13.27 \%$ & $26.99 \%$ \\
\hline & $500 \mathrm{~m}$ & $6.22 \%$ & $12.30 \%$ & $24.41 \%$ & $6.83 \%$ & $13.71 \%$ & $27.39 \%$ \\
\hline
\end{tabular}

The Selected Links cases have in general higher average percentages of valid connected vehicles than the Whole Network cases because more equipped vehicles are on selected freeways and major arterials and they can communicate with one another more often. The actual market penetration rates of equipped vehicles in the Selected Links cases are also higher than those in the Whole Network cases because more vehicles are on freeways and major arterials than on minor arterials, local, and collector roads. About $77.5 \%$ of total vehicles are 
on these selected links in this study. With this difference in market penetration rate, it's difficult to directly compare the results of the average percentages of valid connected vehicles between the Whole Network and the Selected Links cases.

The average success rate of equipped vehicles to communicate with other equipped vehicles in the study area is shown in Table 2. At least $90 \%$ of equipped vehicles are able to communicate with nearby equipped vehicles in Whole Network case. Most equipped vehicles, more than $99.5 \%$, can communicate with nearby equipped vehicles when wireless communication coverage is 500 meters in Whole Network case. These average success rates of communication in the Selected Links case are lower than those in the Whole Network case because equipped vehicles under the Selected Links case cannot communicate with other equipped vehicles travelling on unselected links such as minor arterials, local streets, and collector roads. But, this difference between the two cases decreases as the percent of market penetration rate increases.

Table 2: Average success rate* of communication among equipped vehicles.

\begin{tabular}{|l|c|c|c|c|c|c|c|}
\hline \multirow{2}{*}{} & \multicolumn{6}{|c|}{ Study area } \\
\cline { 2 - 8 } & \multicolumn{3}{|c|}{ Whole Network } & \multicolumn{3}{c|}{ Selected Links } \\
\hline $\begin{array}{l}\text { Market penetration rate of } \\
\text { equipped vehicles }\end{array}$ & $10 \%$ & $20 \%$ & $40 \%$ & $10 \%$ & $20 \%$ & $40 \%$ \\
\hline $\begin{array}{l}\text { Wireless } \\
\begin{array}{l}\text { Communication } \\
\text { Coverage }\end{array}\end{array}$ & $100 \mathrm{~m}$ & $90.08 \%$ & $95.31 \%$ & $97.79 \%$ & $76.89 \%$ & $89.17 \%$ & $94.70 \%$ \\
\cline { 2 - 8 } & $500 \mathrm{~m}$ & $96.65 \%$ & & & & & \\
\cline { 2 - 8 }
\end{tabular}

*Average success rate of communication $=$ (number of valid connected vehicles/number of equipped vehicles) $* 100$.

\subsection{Spatial-temporal dispersion of equipped vehicles}

The area coverage level of valid connected vehicle is used to assess the spatialtemporal dispersion of equipped vehicles. The concept of area coverage level adopted in this study is different from the ones used in previous studies that are discussed in the literature review. The cumulative fraction of number of covered links by probe vehicles was used in previous studies. Also, if one probe vehicle is on a certain link, this link was assumed to be covered to estimate travel time or speed. In addition, previous studies only considered short time durations such as 5,10 , and 15 minutes and low penetration rate of probe vehicles such as $5 \%$. This study uses the average fraction of links covered by platoons of valid connected vehicles over total lengths of all links in the study area at every 1 minute during 4 hours.

Table 3 shows the average percentage of the study area covered by valid communication of equipped vehicles for the $3 \times 3$ scenarios of market penetration rate and communication coverage. The average area coverage level is the proportion between the linear lengths of links covered by valid connected vehicles and the total linear lengths of all links in the study area. As shown in Table 3, all average area coverage levels on the Selected Links case are higher 
than those of the Whole Network cases because about $77.5 \%$ of total vehicles are on these selected links. Moreover, the total lengths of selected links also are much smaller than the total lengths of all links in the study area. The average area coverage level at every 15 minute interval during 4 hours (6-10 a.m.) is presented to investigate variations of area coverage level in Figure 3 for one scenario having $20 \%$ of market penetration rate and $200 \mathrm{~m}$ of wireless communication coverage. This Figure indicates that the average area coverage varies by time of day and is sensitive to the density of traffic. It shows that the area coverage could be as high as $22.19 \%$ at $7: 30$ a.m. and as low as $0.28 \%$ at 10:00 a.m. on the Selected Links network.

Table 3: Average area coverage level* of valid connected vehicles.

\begin{tabular}{|l|c|c|c|c|c|c|c|}
\hline \multirow{2}{*}{} & \multicolumn{6}{|c|}{ Study area } \\
\cline { 2 - 8 } & \multicolumn{3}{|c|}{ Whole Network } & \multicolumn{3}{c|}{ Selected Links } \\
\hline $\begin{array}{l}\text { Market penetration rate of } \\
\text { equipped vehicles }\end{array}$ & $10 \%$ & $20 \%$ & $40 \%$ & $10 \%$ & $20 \%$ & $40 \%$ \\
\hline & $100 \mathrm{~m}$ & $1.39 \%$ & $4.08 \%$ & $8.43 \%$ & & & \\
$\begin{array}{l}\text { Wireless } \\
\begin{array}{l}\text { Communication } \\
\text { Coverage }\end{array}\end{array}$ & $200 \mathrm{~m}$ & $2.37 \%$ & $5.83 \%$ & $10.96 \%$ & & & \\
\cline { 2 - 9 } & $500 \mathrm{~m}$ & $3.38 \%$ & $7.29 \%$ & $12.27 \%$ & $7.29 \%$ & $16.60 \%$ & $30.33 \%$ \\
\hline
\end{tabular}

*Average area coverage level $=($ Linear lengths of highways covered by valid connected vehicles/total linear lengths of highways in the study area) $* 100$.
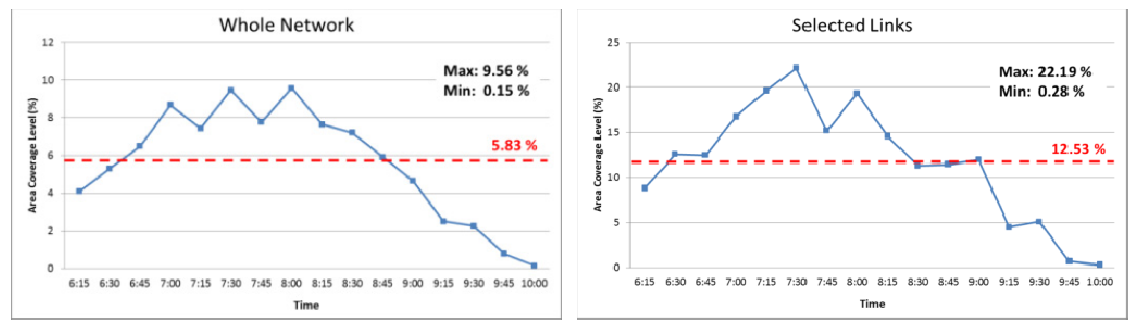

Figure 3: Average area coverage level for 15 min interval.

\subsection{Spatial-temporal dispersions of equipped vehicles using a 10 seconds time interval}

Instead of the one minute update interval that was presented earlier, the authors investigated the spatial-temporal distribution of equipped vehicles using 10 seconds interval for a 30-minute period during peak traffic volumes from 7:45 a.m. to 8:15 a.m. The 10 seconds update interval would produce higher density of vehicular traffic which is expected to produce higher percentage of valid 
communications among the equipped vehicles. One scenario having $20 \%$ of market penetration rate and $200 \mathrm{~m}$ of wireless communication coverage was analyzed for this case. The average area coverage levels of valid connected vehicles in the Whole Network and the Selected Links cases are 8.84 and $18.81 \%$ respectively in Table 4 . Both average percentage of valid connected vehicles and spatial-temporal dispersion results of equipped vehicles during 30 minutes (from 7:45 to 8:15 a.m.) at every 10 seconds are higher than the previous results obtained during 4 hours (from 6:00 to 10:00 a.m.) as shown in Table 4. Especially, average area coverage levels in both Whole Network and Selected Links cases are increased by 50\% compared with the previous results from 4 hours analysis. Because, this 30 minutes period provided a much higher density of traffic. As the traffic density increases, more equipped vehicles are able to communicate with one another.

Table 4: Average percentage and average area coverage level of valid connected vehicles for 10 seconds interval during 30 minutes.

\begin{tabular}{|l|c|c|}
\hline Study area & $\begin{array}{c}\text { Average percentage of } \\
\text { valid connected vehicles }\end{array}$ & Average area coverage level \\
\hline Whole Network & $13.23 \%\left(+9.16 \% \%^{*}\right)$ & $8.84 \%(+51.63 \% *)$ \\
\hline Selected Links & $14.40 \%(+8.52 \% *)$ & $18.81 \%\left(+50.12 \%^{*}\right)$ \\
\hline
\end{tabular}

*Percent difference with results from 1- minute update and 4 hours analysis presented in Sections 5.1 and 5.2

\section{Conclusions}

The success rate of wireless communication and spatial-temporal dispersion for $\mathrm{V} 2 \mathrm{~V}$ are investigated based on the market penetration rate of equipped vehicles and wireless communication coverage in the realistic and large network. Both average success rate and area coverage level increase as the market penetration rate and wireless communication coverage increase. At least $90 \%$ of equipped vehicles success to communicate with nearby equipped vehicles in Whole Network case. All Selected Links cases have lower average success rates of communication than Whole Network cases because equipped vehicles on the selected links cannot communicate with other equipped vehicles travelling on unselected links. The average area coverage level is used to assess the spatialtemporal dispersion of equipped vehicles. The market penetration rate of equipped vehicles has a greater influence on spatial-temporal dispersion of equipped vehicles. The Selected Links case has higher average area coverage level than the Whole Network case although it has lower success rate of communication. This is because $77.5 \%$ of total vehicles are on these selected links and the total lengths of selected links also are much smaller than the total lengths of all links in the study area. The average area coverage varies by time of day and is sensitive to the density of traffic. Finally, the spatial-temporal dispersion of equipped vehicles increase as the density of traffic increases, i.e. more equipped vehicles are able to communicate with one another. These study 
findings will be useful for making decisions about investments in cooperative vehicles.

The distance of information propagation from one and multiple information sources through wireless multi-hop will be investigated as future works. Equipped vehicles' owners from a socio-economic aspect and the communication duration rate of equipped vehicles also will be considered. Sufficient level of connected vehicle applications deployment will be determined for reliable traffic management.

\section{References}

[1] Brian Gallagher, Hidehiko Akatsuka, and Hideaki Suzuki. Wireless Communications for Vehicle Safety: Radio Link Performance \& Wireless Connectivity Methods. Convergence Transportation Electronics Association and SAE International, 2006.

[2] R. Meireles, M. Boban, P. Steenkiste, O. Tonguz, and J. Barros. Experimental Study on the Impact of Vehicular Obstructions in VANETs. IEEE Vehicular Networking Conference, 2010.

[3] ASTM E2213-03 - Standard Specification for Telecommunications and Information Exchange Between Roadside and Vehicle Systems $-5 \mathrm{GHz}$ Band Dedicated Short Range Communications (DSRC) Medium Access Control (MAC) and Physical Layer (PHY) Specifications. www.standards.its.dot.gov/fact_sheetp.asp? $\mathrm{f}=66$.

[4] IEEE 1609 - Family of Standards for Wireless Access in Vehicular Environments (WAVE). www.standards.its.dot.gov/fact_sheet.asp? $\mathrm{f}=80$.

[5] Steven E. Shladover, Gungor Polatkan, Raja Sengupta, Joel VanderWerf, Mustafa Ergen, and Benedicte Bougler. Dependence of Cooperative Vehicle System Performance on Market Penetration. Transportation Research Record: Journal of the Transportation Research Board, No. 2000, Transportation Research Board of the National Academies, Washington, D.C., pp. 121-127, 2007.

[6] Cheol Oh, Taehyung Kim, Wonkyu Kim, Seungpyo Hong, and Junhyeong Park. Capability-Enhanced Probe Vehicle Surveillance System with Vehicle-to-Vehicle Communications. Transportation Research Record: Journal of the Transportation Research Board, No. 2189, Transportation Research Board of the National Academies, Washington, D.C., pp. 8-16, 2010.

[7] Bing Mei, Hyejung Hu, Nagui M. Rouphail, and Jae-Joon Lee. Simulation Model for Studying Impact of Vehicle-to-Vehicle Wireless Communications on Traffic Network Operations. Transportation Research Record: Journal of the Transportation Research Board, No. 2189, Transportation Research Board of the National Academies, Washington, D.C., pp. 107-115, 2010.

[8] Byungkyu Park and Joyoung Lee. Assessing Sustainability Impacts of Route Guidance System under Cooperative Vehicle Infrastructure 
Environment. Proceedings of the 2009 IEEE International Symposium on Sustainable Systems and Technology. pp. 1-6, 2009.

[9] Ygnace, J. L., C. Drane, Y. B. Yim, and R. de Lacvivier. Travel Time Estimation on the San Francisco Bay Area Network Using Cellular Phones as Probes. UCB-ITS-PWP-2000-18. California PATH, Richmond, 2000.

[10] Karthik K. Srinivasan and Paul P. Jovanis. Determination of Number of Probe Vehicles Required for Reliable Travel Time Measurement in Urban Network. Transportation Research Record 1517, pp. 15-22, 1996.

[11] Susanne Breitenberger, Bernhard Grüber, Martina Neuherz and Ronald Kates. Traffic information potential and necessary penetration rates. BMW Group, 2004.

[12] Turner, S. and D. Holdener. Probe Vehicle Sample Sizes for Real-Time Information: The Houston Experience. Proceedings of the Vehicle Navigation and Information Systems Conference, Seattle, Wash., IEEE, pp. 3-10, 1995.

[13] Matthew W. Green, Michael D. Fontaine, and Brian L. Smith. Investigation of Dynamic Probe Sample Requirements for Traffic Condition Monitoring. Transportation Research Record: Journal of the Transportation Research Board, No. 1870, TRB, National Research Council, Washington, D.C., pp. 55-61, 2004.

[14] A.G. Hobeika, et.al.. TRANSIMS - The Population Synthesizer Module, A formal report submitted to FHWA, Washington, D.C, 2001.

[15] K.S. Lee, and A.G. Hobeika. Application of Dynamic Value Pricing Through Enhancements to "TRANSIMS" Journal of the Transportation Research Board No. 2003, pp. 7-16, 2007. 